\title{
Um Agente Pedagógico Gentil é Mais Efetivo? Efeito das Atitudes de Agentes Pedagógicos Animados na Aprendizagem, Engajamento, Emoções e Ansiedade dos Estudantes
}

\author{
Otávio B. Azevedo ${ }^{1}$, Patricia A. Jaques ${ }^{1}$ \\ ${ }^{1}$ Programa de Pós-Graduação em Computação Aplicada (PPGCA) \\ Universidade do Vale do Rio dos Sinos (UNISINOS), São Leopoldo - RS - Brasil \\ otavioazevedo@outlook.com.br, pjaques@unisinos.br
}

\begin{abstract}
The interest in animated agents with affective dimensions has increased considerably in recent years. However, there was no comparison of the effects of animated pedagogical agents with opposite attitudes (such as friendly and direct, for example) and neutral (without emotions). Thus, the objective of this work is to verify the impact of the agents attitudes on students in relation to learning, engagement, emotions and state of anxiety. To achieve this goal, an agent with friendly, direct and neutral attitudes was developed and integrated with the PAT2Math. This system was used in an experimental evaluation involving 71 students of the seventh grade of elementary school, which were distributed in three groups, one for each agent's attitude. The results indicated that the impact of the friendly agent on students was more positive than other attitudes.
\end{abstract}

Resumo. O interesse pelos agentes animados com dimensões afetivas tem aumentado consideravelmente nos últimos anos. Entretanto, não houve uma comparação dos efeitos de agentes pedagógicos animados com atitudes opostas (como amigável e direta, por exemplo) e neutras (sem emoções). Dessa maneira, o objetivo deste trabalho é verificar o impacto das atitudes dos agentes nos alunos em relação à aprendizagem, engajamento, emoções e estado de ansiedade. Para alcançar este objetivo, uma agente com as atitudes amigável, direta e neutra foi desenvolvida e integrada ao PAT2Math. Esse sistema foi utilizado em uma avaliação experimental envolvendo 71 alunos do sétimo ano do ensino fundamental, os quais foram distribuídos em três grupos, um para cada atitude da agente. Os resultados indicaram que o impacto da agente amigável nos alunos foi mais positivo em relação às outras atitudes.

\section{Introdução}

Uma boa comunicação entre o professor e o aluno é fundamental para que ocorra aprendizagem, seja presencial ou à distância [Morreale and Pearson 2008]. Esse princípio também é válido para os Sistemas Tutores Inteligentes, que geralmente se comunicam com o estudante através de texto e/ou áudio. No entanto, os alunos podem sentir falta da presença do professor, de maneira que a comunicação não seja tão eficaz como em uma aula presencial. Os Agentes Pedagógicos Animados (APAs) podem ajudar a minimizar este problema, trazendo uma representação virtual do professor que pode se comunicar com o estudante pela fala, gestos e expressões faciais humanas [Jaques and Vicari 2005]. 
Estudos mostraram que os APAs se tornam mais eficientes quando estabelecem uma relação afetiva com os seus alunos como se fossem um tutor humano, fazendo com que eles aprendam mais [Jaques et al. 2008, Karacora et al. 2012]. Segundo [Scherer 2005], a postura afetiva adotada por um indivíduo em relação ao outro em uma determinada situação é denominada postura interpessoal. Entretanto, no contexto dos Agentes Pedagógicos, essa é a definição de atitude [Ferguson 1992]. Como este trabalho está na área dos APAs, o termo atitude foi utilizado ao invés de postura interpessoal.

Existem uma série de trabalhos que buscaram verificar a eficiência de APAs com atitudes empática, polida, rude e com rapport ${ }^{1}$ no aumento da aprendizagem e engajamento dos alunos [Jucks et al. 2018, Hastie et al. 2016, Krämer et al. 2016], e alguns estudos também analisaram o impacto desses agentes nos estados afetivos dos alunos [Moridis and Economides 2012, Wang et al. 2010]. Entretanto, esses trabalhos não compararam os efeitos de agentes pedagógicos animados com atitudes opostas (como a educação e a rudeza, por exemplo) e neutras (sem emoções), e não consideraram o estado de ansiedade dos estudantes. Nesse contexto, o objetivo geral deste trabalho é verificar o impacto das atitudes dos agentes pedagógicos animados nos alunos em relação à aprendizagem, engajamento, emoções e estado de ansiedade.

Para alcançar esse objetivo, uma agente pedagógica animada com atitudes (podendo ser amigável, direta ou neutra) foi desenvolvida e integrada ao PAT2Math para fins de avaliação. O sistema foi utilizado por 71 alunos do sétimo do ensino fundamental durante 10 semanas. Os alunos foram distribuídos aleatoriamente em três grupos, sendo um grupo para cada atitude da agente.

O PAT2Math é um Sistema Tutor Inteligente baseado em passos que assiste os estudantes a resolverem equações algébricas de primeiro grau com uma incógnita [Jaques et al. 2013].

Os Sistemas Tutores Inteligentes (STIs) são programas computacionais destinados a auxiliar os alunos no processo de ensino-aprendizagem, utilizando técnicas de inteligência artificial e teorias pedagógicas para adaptar-se às características desses estudantes, proporcionando assim um aprendizado individualizado [Jaques 2007]. Ele fornece um feedback mínimo para cada um dos passos resolvidos, e uma mensagem de ajuda em caso de erro. Os alunos também podem pedir ajuda caso não saibam como prosseguir na resolução da equação.

\section{Trabalho Desenvolvido}

A agente desenvolvida neste trabalho conta com atitudes afetivas e foi integrada ao PAT2Math. Ela expressa a sua atitude a partir da fala, velocidade de voz e expressões faciais, e tem como objetivo auxiliar os alunos na resolução de equações de primeiro grau pela interface do sistema. Esse suporte pode ser de duas formas:

1. Dicas: podem ser solicitadas a qualquer momento pelo aluno durante a resolução de um exercício;

2. Feedbacks de erro: são disponibilizados quando o aluno erra um passo na equação. Na maioria das vezes esses feedbacks informam para o estudante o que

\footnotetext{
${ }^{1}$ Este termo em inglês é empregado na área da psicologia para definir uma relação de sincronia e empatia entre duas ou mais pessoas, o qual não possui uma tradução específica para o português.
} 
está errado e fornecem uma dica para corrigir o erro. Porém, se o sistema não identificar com precisão o erro cometido pelo aluno, a Janet fornece um feedback mais genérico.

Além da agente e do sistema de ajuda e feedback, foi elaborado um roteiro de exercícios que os alunos resolveram durante o experimento. Os detalhes dessas três atividades realizadas estão descritos a seguir.

\subsection{Agente Pedagógico Animado}

A agente recebeu o nome de Janet e foi desenvolvida a partir das linguagens de programação e marcação WEB: HTML5, CSS e JavaScript. As animações foram realizadas a partir de imagens GIF e o texto das falas era exibido na parte inferior da página, como se fosse uma legenda.

Além dos recursos visuais, a Janet possui um sistema de áudio, que converte o texto exibido na tela em voz. Esta funcionalidade foi implementada a partir de uma API nativa dos navegadores de internet, denominada Speech Synthesis. A Speech Synthesis disponibiliza uma lista de vozes em uma grande variedade de idiomas, incluindo o português brasileiro. Além disso, a API permite configurar a velocidade da voz e a sua frequência.

A agente está disponível em três versões, as quais são baseadas em duas atitudes opostas (amigável e direta ${ }^{2}$ ) e uma neutra. Todas as versões possuem a mesma aparência física, mas diferem em três atributos: expressões faciais, velocidade de voz e estilo de fala. A Tabela 1 apresenta as diferenças entre as versões e o vídeo a seguir mostra a Janet amigável em funcionamento no PAT2Math na perspectiva de cada atitude: https: //youtu.be/eBcG0RHvfto

\begin{tabular}{|c|c|c|}
\hline Amigável & Direta & Neutra \\
\hline $\begin{array}{c}\text { Alto nível de educação e } \\
\text { polidez }\end{array}$ & $\begin{array}{l}\text { Falas e textos mais } \\
\text { diretos, sem polidez }\end{array}$ & \multirow{4}{*}{ Sem interações afetivas } \\
\hline $\begin{array}{l}\text { Incentiva o aluno a pedir } \\
\text { ajuda quando sente } \\
\text { dificuldades }\end{array}$ & $\begin{array}{l}\text { Chama a atenção do aluno } \\
\text { se ele pedir ajuda mais de } \\
\text { três vezes seguidas }\end{array}$ & \\
\hline $\begin{array}{c}\text { Conforta o aluno em caso } \\
\text { de erro }\end{array}$ & $\begin{array}{l}\text { Repreende o aluno em } \\
\text { caso de erro }\end{array}$ & \\
\hline $\begin{array}{l}\text { Cumprimenta o aluno } \\
\text { após o login no sistema de } \\
\text { uma forma mais simpática }\end{array}$ & $\begin{array}{l}\text { Cumprimenta o aluno } \\
\text { após o login no sistema de } \\
\text { uma forma mais direta }\end{array}$ & \\
\hline Velocidade de voz normal & $\begin{array}{l}\text { Velocidade de voz um } \\
\text { pouco rápida }\end{array}$ & Velocidade de voz rápida \\
\hline
\end{tabular}

Tabela 1. Comparação das Três Versões da Janet

\footnotetext{
${ }^{2}$ A ideia original seria considerar a atitude rude no lugar da direta, que seria o nível máximo de oposição à amigável. Entretanto, essa ideia foi abandonada porque ela poderia trazer efeitos negativos para os alunos.
} 


\subsection{Sistema de Ajuda e Feedback}

Anteriormente, PAT2Math contava com um sistema de dicas baseado em cinco níveis, sendo que os dois primeiros oferecem dicas mais genéricas e que exigem um maior raciocínio, o terceiro e o quarto mostram dicas mais pontuais e o quinto nível indica a resposta do próximo passo ao aluno.

O tutor também fornece feedbacks de erro com três níveis, que auxiliam o aluno a corrigir o passo errado no sistema. Assim como nas dicas, quanto maior o nível, mais específico é o feedback, e o último nível revela a resposta do próximo passo ao estudante.

O sistema desenvolvido segue a mesma técnica de divisão por níveis descrita anteriormente, mas agora eles estão escritos em uma linguagem mais informal e acessível para os alunos. Esse sistema foi implementado de forma genérica, podendo ser utilizado para quaisquer equações de primeiro grau com uma incógnita. A única limitação é para as equações que possuem frações com a incógnita no denominador. Mas se a incógnita estiver no numerador, o sistema funcionará corretamente. A tabela que lista todas as possibilidades de dicas e feedbacks de erro está disponível em: https: //tinyurl.com/dicas-e-feedbacks

\subsection{Roteiro de Exercícios}

Os exercícios que os alunos resolveram durante o experimento foram elaborados com o auxílio de duas professores de matemática. As equações foram distribuídas em sete planos de aula, sendo que o primeiro possui equações no formato $a x+b=c$ e com vários termos, pois os alunos já aprenderam os conteúdos básicos das equações e muito deles já estavam avançados no tutor. O motivo da criação de novos exercícios é para que todos os estudantes pudessem começar do mesmo ponto, tornando a análise mais completa dos progressos deles durante a avaliação. A Tabela 2 possui a lista completa dos planos de aula, bem como suas descrições e exemplos de equações.

\begin{tabular}{|c|c|c|}
\hline Plano & Descrição & Exemplo de equação \\
\hline 1 & $\begin{array}{c}\text { Revisão geral, com equações no } \\
\text { formato } a x+b=c \text { com vários termos }\end{array}$ & $4 x+2 x=42-10 x$ \\
\hline 2 & $\begin{array}{l}\text { Equações que envolvem adição, } \\
\text { subtração e multiplicação de números e } \\
\text { termos entre parênteses }\end{array}$ & $5 x-(x-2)+4(3 x+8)=10$ \\
\hline 3 & Razão e proporção & $\frac{4 x}{2}=\frac{x+10}{5}$ \\
\hline 4 & $\begin{array}{c}\text { Frações simples com denominadores } \\
\text { diferentes que exigem o cálculo do } \\
\text { Mínimo Múltiplo Comum }\end{array}$ & $\frac{x}{4}+\frac{2 x}{5}-\frac{5}{2}=42$ \\
\hline 5 & Frações com numeradores compostos & $\frac{x+2}{8}+\frac{x}{4}-\frac{2 x-6}{8}=12$ \\
\hline 6 & Frações com propriedade distributiva & $\frac{3(2 x-5)}{4}+\frac{5 x}{2}=20$ \\
\hline 7 & $\begin{array}{c}\text { Desafio com as equações mais difíceis } \\
\text { que misturam todos os conteúdos } \\
\text { aprendidos }\end{array}$ & $-\frac{121 x}{11}=\frac{385 x}{55}-22(11+22 x)$ \\
\hline
\end{tabular}

Tabela 2. Lista de Planos de Aula Elaborados Para o Experimento 


\section{Avaliação: Método}

O experimento teve uma duração total de 10 semanas, nas quais os alunos utilizaram o sistema desenvolvido no laboratório de informática do colégio durante um período de 50 minutos. Participaram 71 estudantes do sétimo ano do ensino fundamental, sendo 37 meninas e 34 meninos, com idades de 12 a 14 anos (média de 12,7 anos e desvio padrão de 0,597$)$. Esses alunos foram distribuídos aleatoriamente entre os três grupos do experimento (agente amigável, direta e neutra).

Em relação à coleta de dados, foram utilizados três diferentes materiais:

1. Testes de Conhecimento: tiveram como objetivo medir o nível de conhecimento dos alunos no conteúdo de equações. Cada teste foi composto por 12 equações e o nível de dificuldade aumentava gradualmente a cada equação;

2. Interações dos usuários no sistema: foram considerados o total de equações resolvidas e o número de vezes que a Janet foi desativada;

3. Questionários: continham perguntas sobre os dados pessoais básicos dos alunos, além do seu perfil, emoções e estado de ansiedade. As emoções e o traço de ansiedade dos alunos foram mensuradas a partir de suas respostas no Inventário De Ansiedade Traço-Estado (IDATE), que corresponde a um questionário validado na literatura [Spielberger et al. 1979]. Os alunos também avaliaram as características funcionais da Janet através de um questionário desenvolvido para esse trabalho.

A avaliação foi dividida em duas partes de mesma duração, sendo que no final da primeira parte ocorreu uma troca nas atitudes amigável e direta: os alunos que estavam com a agente amigável passaram a utilizar a agente direta e vice-versa (os alunos do grupo controle permaneceram utilizando a agente neutra).

A Figura 1 apresenta o desenho do experimento. $\mathrm{Na} 1^{\text {a }}$ semana os alunos resolveram o pré-teste de conhecimento. Na semana seguinte foi realizado um teste piloto do sistema. Entre a $3^{\mathrm{a}}$ e a $5^{\mathrm{a}}$ semana ocorreu a primeira parte da avaliação, sendo que nos 15 minutos finais da quinta sessão os alunos responderam ao questionário referente às três semanas de utilização do sistema.

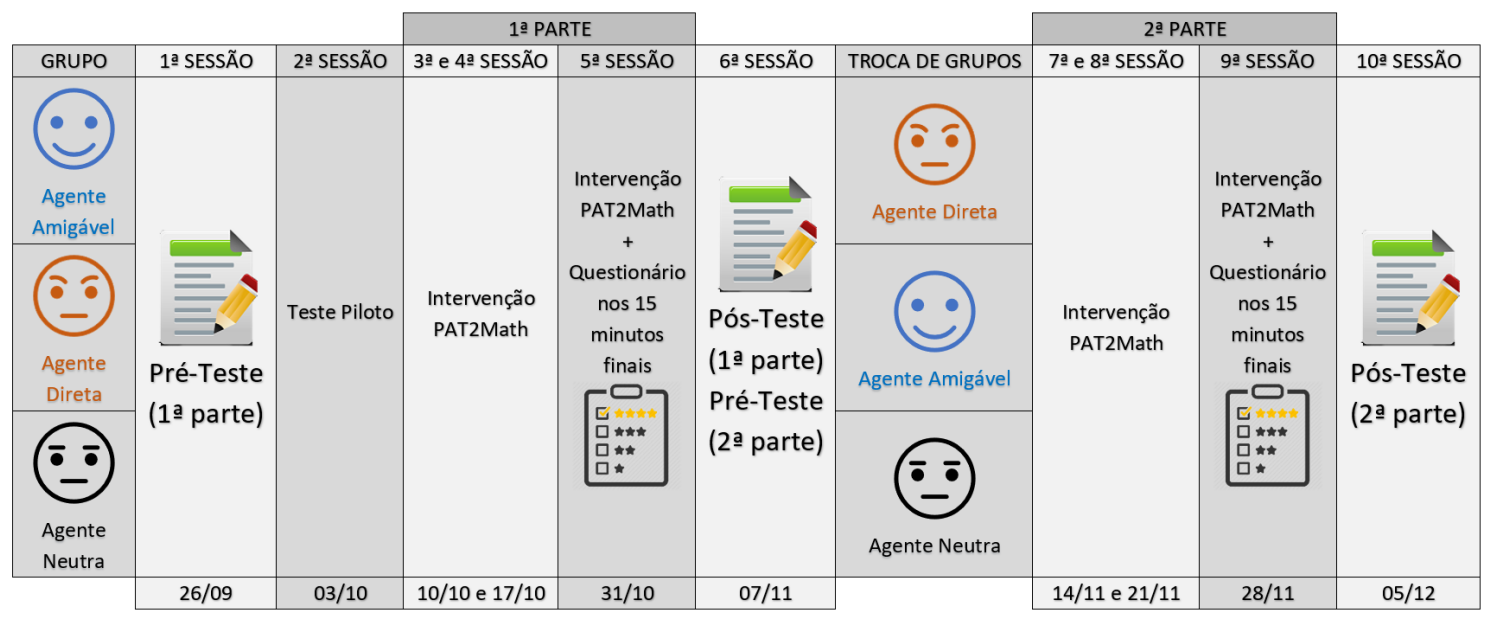

Figura 1. Desenho do Experimento

$\mathrm{Na} 6^{\mathrm{a}}$ semana os alunos responderam ao pós-teste da primeira parte da avaliação, que também serviu como pré-teste para a segunda parte. Na $7^{\mathrm{a}}$ semana ocorreu a troca 
das atitudes amigável e direta, que marcou o início da segunda parte do experimento. Essa segunda parte teve sua conclusão na $9^{a}$ semana, na qual os alunos responderam ao questionário nos 15 minutos finais. Finalmente, na $10^{\mathrm{a}}$ semana, os alunos responderam ao pós-teste da segunda parte do experimento.

\section{Análise e Discussão dos Resultados}

Os resultados da avaliação experimental realizada com a agente estão descritos nesta seção, a qual foi dividida em cinco partes: as quatro primeiras correspondem à validação do objetivo deste trabalho e a última parte descreve as limitações do experimento.

\subsection{Aprendizagem}

A Figura 2 contêm os gráficos com as notas que os alunos conseguiram nos testes de conhecimento nas duas etapas da avaliação. Os ganhos de aprendizado foram calculados a partir da diferença entre a nota do pós-teste pela nota do pré-teste.
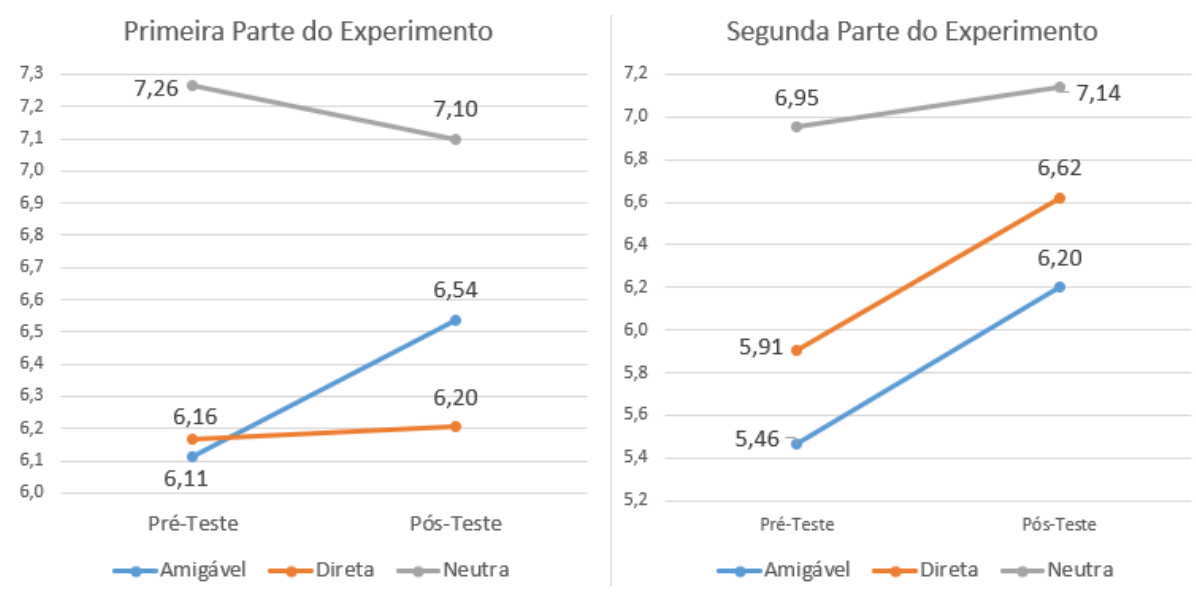

Figura 2. Resultados dos Testes de Conhecimento

Observa-se que em ambas as partes do experimento os alunos que utilizaram a Janet amigável obtiveram um maior ganho de aprendizado em relação aos grupos da Janet direta e neutra. Além disso, os alunos que contaram com a Janet direta apresentaram um melhor desempenho do que o grupo que utilizou a Janet neutra ${ }^{3}$.

Apesar de os gráficos apresentarem diferenças notáveis de ganhos de aprendizado entre os três grupos, não foram encontradas diferenças com significância estatística. Acredita-se que o tamanho pequeno das amostras de alunos que realizaram pré-teste e o pós-teste tiveram um impacto negativo nesses resultados, principalmente na segunda etapa do experimento: vários alunos faltaram no dia do pós-teste, o que resultou em amostras com menos de 15 estudantes.

\subsection{Engajamento}

O nível de engajamento dos alunos foi estimado a partir de duas fontes de dados (1) Questionário sobre a Janet: quanto mais eles gostaram da agente, mais engajados estavam

\footnotetext{
${ }^{3} \mathrm{O}$ grupo neutro apresentou as melhores notas (uma boa parte de seus alunos tinha um maior conhecimento prévio), porém não obteve o maior nível de ganhos de aprendizado.
} 
com a utilização do sistema; (2) Logs no sistema: quanto mais equações eles resolveram e menos vezes desativaram a Janet, mais engajado estavam com o uso do sistema.

Os alunos que utilizaram a Janet amigável registraram avaliações mais positivas nas duas partes do experimento e se mostraram mais engajados em utilizar o sistema com a agente do que os demais grupos ${ }^{4}$. Além disso, ao comparar as avaliações dos grupos das atitudes direta e neutra, verificou-se que a Janet direta teve uma avaliação levemente superior em relação à Janet neutra.

Também foi observado que os estudantes que utilizaram a Janet neutra resolveram mais equações na primeira etapa do experimento, e na segunda parte os alunos do grupo amigável concluíram mais exercícios. Isso pode ter acontecido porque na segunda etapa as equações eram mais complexas e os alunos que utilizaram a Janet amigável se sentiam mais confortáveis para pedir ajuda. Além disso, a Janet amigável foi desativada menos vezes que a direta e a neutra em ambas as partes do experimento, sendo que na primeira nenhum aluno a desativou.

Foram identificadas diferenças com significância estatística nas respostas de duas perguntas em cada parte do experimento, sendo que na segunda parte também foi verificada uma diferença significativa no número de vezes que os alunos desativaram a Janet.

\subsection{Emoções}

As emoções dos alunos foram mensuradas a partir de suas respostas no questionário IDATE-E. Esse questionário possui uma lista de 20 emoções, sendo 10 positivas e 10 negativas. Os estudantes avaliaram a intensidade dessas emoções que estavam sentindo naquele momento, a partir de uma escala de 1 (absolutamente não) a 4 (muitíssimo).

Houve uma maior intensidade de emoções positivas nos alunos que utilizaram a Janet direta na primeira parte do experimento e uma maior intensidade de emoções negativas nos estudantes que contaram com a Janet amigável. Por sua vez, na segunda etapa da avaliação, houve uma maior intensidade de emoções positivas nos estudantes do grupo da Janet amigável e uma menor intensidade nos alunos do grupo da Janet direta. Além disso, os alunos que utilizaram a Janet direta relataram mais emoções negativas do que os demais estudantes.

Um possível motivo para a Janet amigável não ter sido a melhor para promover emoções positivas na primeira parte é devido aos problemas que aconteceram com o sistema de voz da agente, o que comprometeu a sua eficácia na etapa inicial (ver mais detalhes na subseção 4.5).

Em relação à análise estatística, não foram encontradas diferenças significativas em nenhuma das comparações.

\subsection{Estado de Ansiedade}

Para medir o estado de ansiedade foi utilizado o questionário IDATE-E, que retorna um coeficiente que varia de 20 a 80 (quanto maior o coeficiente, maior a ansiedade). Assim sendo, gráfico da Figura 3 apresenta as médias desses coeficientes nas duas etapas do experimento.

\footnotetext{
${ }^{4}$ Os gráficos com as respostas dos alunos nas duas partes do experimento estão disponíveis em: https : //tinyurl.com/questionario-engajamento
} 


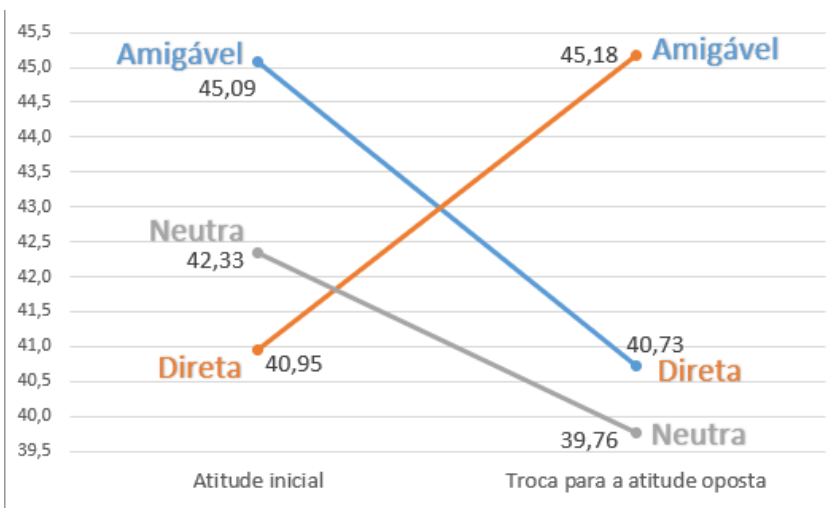

Figura 3. Médias dos Estados de Ansiedade nas Duas Etapas do Experimento

Observa-se que houve uma redução de 4,36 pontos no estado de ansiedade dos alunos do grupo da Janet amigável que passaram a utilizar a Janet direta na segunda etapa do experimento. Por outro lado, no caso dos estudantes que começaram no grupo da Janet direta e passaram a utilizar Janet amigável na segunda parte da avaliação, houve um aumento no estado de ansiedade (4,23 pontos).

Acredita-se que os alunos desejam ser mais performáticos com professores que eles gostam porque desejam agradá-los, o que pode resultar em uma maior ansiedade. Não encontramos outros estudos científicos que encontraram tal relação. Outra explicação possível é que o grupo de estudantes que usou o agente amigável era formado predominantemente por alunos com locus of control externo, ou seja, que acreditam que têm pouco controle sobre seu futuro e, por isso, ficam mais ansiosos com a presença de humanos ou agentes [Rickenberg and Reeves 2000]. No experimento desses autores, foi identificado que os participantes ficaram mais ansiosos quando os agentes os monitoravam durante a utilização do sistema, e esse efeito era mais forte para as pessoas com locus of control externo. É importante ressaltar, no entanto, que desde que a ansiedade não seja muito alta, ela também pode trazer benefícios, como motivar a ação.

Não foram encontradas diferenças com significância estatística na comparação dos estados de ansiedade dos três grupos, apesar de os gráficos indicarem o contrário. Possivelmente o alto desvio padrão das médias dos coeficientes IDATE-E, que apresentaram valores entre 10,6442 e 13,8339, teve uma grande influência nos resultados.

\subsection{Limitações do experimento}

Durante as 10 semanas da avaliação, ocorreram alguns problemas e situações externas que podem ter influenciado de maneira negativa os resultados do experimento. O sistema de voz da Janet só pôde ser utilizado por completo na segunda etapa do experimento devido à problemas de compatibilidade não previstos com os sistemas operacionais dos computadores da escola, os quais rodavam o Windows 7.

Também ocorreu uma situação específica em uma das três turmas do experimento: haviam muitos alunos dispersos e que não fizeram corretamente as atividades em vários momentos da avaliação. Apesar de a distribuição dos alunos entre os grupos ter sido totalmente aleatória, essa turma pode ter impactado nos resultados, uma vez que ela correspondia a $20 \%$ dos participantes do experimento. 
IX Congresso Brasileiro de Informática na Educação (CBIE 2020)

Anais do XXXI Simpósio Brasileiro de Informática na Educação (SBIE 2020)

\section{Conclusão}

Os Agentes Pedagógicos Animados possuem uma melhor eficiência e são mais aceitos quando estabelecem uma relação afetiva com os seus alunos, pois dessa maneira eles se aproximam mais de um tutor humano e interagem de maneira mais antropomórfica [Wang et al. 2010]. Nesse sentido, uma questão aberta é quais as características afetivas do agente são mais determinantes para uma boa relação. Assim, este trabalho propõe uma Agente Pedagógica Animada que possui diferentes atitudes afetivas - amigável, direta e neutra - para os STIs baseados em passos e possui os seguintes objetivos: analisar o efeito dessas atitudes nos alunos em relação ao engajamento, aprendizagem, emoções e estado de ansiedade, além de verificar se os alunos que gostaram mais da agente apresentaram um melhor desempenho na disciplina do que os demais estudantes.

A agente desenvolvida neste trabalho recebeu o nome de Janet e foi integrada ao PAT2Math para fins de avaliação e foi responsável por ajudar os alunos quando eles sentirem dificuldades em um exercício e fornecer feedbacks para os passos registrados no sistema. Essa avaliação contou com a participação de 71 alunos do sétimo ano do ensino fundamental, que usaram três versões do agente: amigável, direta e neutra.

Os resultados indicaram que os alunos do grupo da agente amigável apresentaram um maior engajamento e ganho de aprendizado, confirmando as avaliações de outros trabalhos da área [Jucks et al. 2018, Guo and Goh 2015, McLaren et al. 2011]. Também houve uma maior intensidade de emoções positivas nos alunos que utilizaram as agentes amigável e direta. Além disso, foi identificada uma redução na ansiedade dos alunos do grupo da agente neutra e dos estudantes que utilizaram a agente amigável na primeira etapa do experimento. Entretanto, não foram identificadas diferenças com significância estatística na maioria das comparações, possivelmente por causa do pequeno tamanho da amostra.

Como trabalho futuros, pretende-se realizar um novo experimento com uma amostra maior de participantes, incluindo escolas públicas e privadas para validade externa do estudo. Também planeja-se validar as expressões faciais das três versões da Janet junto a psicólogos e professores, para definir se as expressões atuais são as mais adequadas para as atitudes representadas pela agente. Além disso, deseja-se estudar com mais profundidade a relação entre a ansiedade dos estudantes e as características afetivas da agente.

\section{Agradecimentos}

Este trabalho foi realizado com apoio da CAPES (Projeto STIC-AMSUD), FAPERGS (Processo 17/2551-0001203-8) e CNPq (processo 309218/2017-9 e bolsa de mestrado).

\section{Referências}

Ferguson, I. A. (1992). Touring machines: Autonomous agents with attitudes. Computer, 25(5):51-55.

Guo, Y. R. and Goh, D. H.-L. (2015). Affect in embodied pedagogical agents: Metaanalytic review. Journal of Educational Computing Research, 53(1):124-149.

Hastie, H., Lim, M. Y., Janarthanam, S., Deshmukh, A., Aylett, R., Foster, M. E., and Hall, L. (2016). I remember you! interaction with memory for an empathic virtual robotic tutor. In $A A M A S$, pages 931-939. IFAAMAS. 
Jaques, P. A. (2007). Estudando os Efeitos Pedagógicos de Agentes Animados Emocionais em Sistemas Tutores Inteligentes. In Blikstein, P. and Reis, R., editors, Panorama das Pesquisas em Computação Afetiva aplicada à Educação no Brasil, pages 85-100, Palo Alto, CA. Lemann Center.

Jaques, P. A., Lehmann, M., and Jaques, K. S. F. (2008). Avaliando a efetividade de um agente pedagógico animado emocional. In SBIE, volume 1, pages 145-154.

Jaques, P. A., Seffrin, H., Rubi, G., de Morais, F., Ghilardi, C., Bittencourt, I. I., and Isotani, S. (2013). Rule-based expert systems to support step-by-step guidance in algebraic problem solving: The case of the tutor pat2math. Expert Systems with Applications, 40(14):5456-5465.

Jaques, P. A. and Vicari, R. M. (2005). Estado da arte em ambientes inteligentes de aprendizagem que consideram a afetividade do aluno. Revista informática na educação: teoria \& prática, 8(1):15-38.

Jucks, R., Linnemann, G. A., and Brummernhenrich, B. (2018). Student evaluations of a (rude) spoken dialogue system insights from an experimental study. Advances in Human-Computer Interaction, 2018.

Karacora, B., Dehghani, M., Kramer-Mertens, N., and Gratch, J. (2012). The influence of virtual agents' gender and rapport on enhancing math performance. In Proceedings of the Annual Meeting of the Cognitive Science Society, volume 34.

Krämer, N. C., Karacora, B., Lucas, G., Dehghani, M., Rüther, G., and Gratch, J. (2016). Closing the gender gap in stem with friendly male instructors? Computers \& Education, 99:1-13.

McLaren, B. M., DeLeeuw, K. E., and Mayer, R. E. (2011). A politeness effect in learning with web-based intelligent tutors. International Journal of Human-Computer Studies, 69(1-2):70-79.

Moridis, C. N. and Economides, A. A. (2012). Affective learning: Empathetic agents with emotional facial and tone of voice expressions. IEEE Transactions on Affective Computing, 3(3):260-272.

Morreale, S. P. and Pearson, J. C. (2008). Why communication education is important. Communication Education, 57(2):224-240.

Rickenberg, R. and Reeves, B. (2000). The effects of animated characters on anxiety, task performance, and evaluations of user interfaces. In $\mathrm{CHI}$, pages 49-56.

Scherer, K. R. (2005). What are emotions? and how can they be measured? Social science information, 44(4):695-729.

Spielberger, C. D., Biaggio, A., and Natalício, L. (1979). Inventário de ansiedade traço estado: manual de psicologia aplicada.

Wang, C.-Y., Ke, S.-Y., Chuang, H.-C., Tseng, H.-Y., and Chen, G.-D. (2010). E-learning system design with humor and empathy interaction by virtual human to improve students' learning. In ICCE, pages 615-622. 